\title{
Threshold Laws for the Break-up of Atomic Particles into Several Charged Fragments
}

\author{
M. Yu. Kuchiev and V. N. Ostrovsky甘 \\ School of Physics, University of New South Wales, Sydney 2052, Australia
}

\begin{abstract}
The processes with three or more charged particles in the final state exhibit particular threshold behavior, as inferred by the famous Wannier law for $(2 e+$ ion) system. We formulate a general solution which determines the threshold behavior of the cross section for multiple fragmentation. Applications to several systems of particular importance with three, four and five leptons (electrons and positrons) in the field of charged core; and two pairs of identical particles with opposite charges are presented. New threshold exponents for these systems are predicted, while some previously suggested threshold laws are revised.
\end{abstract}

PACS numbers: $32.80 . \mathrm{Fb}, 34.80 . \mathrm{Dp}, 34.80 . \mathrm{Kw}$ 


\section{INTRODUCTION}

The famous Wannier [1] threshold law has quite an unusual status among other threshold laws in quantum mechanics. Being based on an appealing mechanism, it has inspired a large number of studies where the law was rederived, extended, tested or rebutted. The intensity of these studies does not show a decrease with time as testify some representative references to the recent publications [2] [3] [4] [5] [6] [7] [8] [9] [10] [11] [12] [13] [14] [15] [16] [17] [18] [19] [20] [21]. A complete bibliography on the subject would be immense.

In this paper we suggest a method which generalizes the Wannier mechanism when break-up of the quantum system on a large number (four or more) of charged fragments is concerned. Apparently, for the first time the particular case of the problem was treated in 1976 in the important paper by Klar and Schlecht [22] where the threshold law was derived for escape of three electrons from the charged core. It was suggested that the receding electrons form a symmetrical configuration of equilateral triangle with the positively charged core in its center. The treatment was quite involving and specialized being based on hyperspherical coordinate system [23]. Later Grujić [24] rederived the same result using the standard Cartesian coordinates where the symmetry considerations are easy to apply explicitly in full

extent. Grujić considered also some other systems along the same lines [25] 26] [27] (see more details in Sec. $\nabla)$. The threshold law for the three-electron escape seems to find support in the experimental data on the near-threshold double ionization of atoms by electron impact [28]. Later Feagin and Filipczyk [29] claimed an existence of a complementary law which is manifested at energies somewhat above a threshold, see critical discussion in Section $\mathrm{V} A$.

The interest to the problem was renewed recently when two electrons and positron receding from the core with $Z=1$ charge were considered by Poelstra et al [30]. A brief note by Stevens and Feagin [31] on complete fragmentation of $\mathrm{H}_{2}$ molecule is also to be mentioned. The final state in the reaction with a positron could be produced by double ionization of a negative ion by positron impact. However, the forthcoming experiments by Knudsen and co-workers 32 concern positron impact double ionization of neutral atoms 
$(Z=2)$ where the threshold law has not yet been available. Its derivation was one of motivations for the present study. Eventually it has developed into a general approach to the multi-fragmentation problem which possesses two important advantages. Firstly, our method describes a general situation with arbitrary number of charged fragments in simple terms in an arbitrary coordinate frame. Secondly, it is convenient and reliable for practical realizations. This allows us to clarify important conceptual aspects of the problem which were misunderstood or misinterpreted previously. Comparing our solution with known in literature results we reproduce a number of threshold exponents for different systems. At the same time we find that several published previously results need improvement, in particular, we revise the threshold law for $2 e^{-}+e^{+}$escape. A general nature of the developed method is illustrated by consideration of new complicated situations with up to six charged particles in the final state where a number of new threshold exponents is predicted.

In Section II we introduce particular configurations which will be called scaling configurations. They describe a multidimensional dynamic potential saddle, generalizing the Wannier ridge which is well known for $(2 e+$ ion $)$ system. These configurations are related to rectilinear trajectories of all particles in the system and play a crucial role for the complete fragmentation process close to its threshold. SCs embrace the essence of previous treatments of particular systems, but avoid attachment to some special theoretical formalism and related technical complications. The closest analogue of our general approach in particular case of three-body Coulomb systems could be found in papers by Simonović and Grujić [33.

Description of small deviations from $\mathrm{SC}$ is given in terms of a set of harmonic oscillators and inverted oscillators (Section [III). The later ones describe unstable modes which govern the threshold law. They are quantized following a general scheme suggested by Kazansky and Ostrovsky 34 [35]. This allows us to construct a reduced form of the wave function for the system of charged particles and derive the threshold law in Section IV generalizing a procedure used previously by Kazansky and Ostrovsky [4] [12] for derivation of the conventional Wannier law. Application of a developed general scheme to some particular systems (Section V) is followed by concluding discussion of special features of the Wannier- 
type threshold laws, with an emphasis on a relation between the underlying statistical and dynamical aspects of the problem (Section VI).

\section{SCALING EXPANSION}

Our goal is to consider some atomic process which breaks an atomic particle into several charged fragments for low excess energy $E$. In this situation the motion of the fragments in the final state of the reaction can be described in the semiclassical approximation because a typical variation of the Coulomb potential $U_{C} \sim 1 / r$ on the wavelengths of the fragments $\lambda \sim 1 / \sqrt{M E}, \delta U \simeq \lambda / r^{2}$, is less than a typical kinetic energy $T \sim E$

$$
\delta U \ll T
$$

inside the Coulomb zone $r \leq r_{C}=1 / E$ where the major events take place. Therefore the first thing to do is to find classical trajectories which lead to the desired final state with total fragmentation.

It is very important that for low energy $E$ there exists a severe restriction onto these trajectories. To see this let us imagine what is happening with distances separating fragments when they move out of the reaction domain. If a distance separating some pair of two attracting fragments diminishes with time, then one should expect that this pair of fragments can be considered as a dipole which interacts with the rest fragments. This interaction can transfer the kinetic energy of the two fragments to the other fragments. Therefore one has to expect that eventually these two fragments will loose enough energy and form a bound state. If this event happens then the desired total fragmentation is not achieved. This discussion shows that one should look for those trajectories which exhibit a monotonic increase of distances separating the fragments. The point is that the lower is the available above-threshold energy the more restrictive this condition is.

It is convenient to present the discussed situation considering the potential energy in the

multidimensional configuration space where its behavior can be described as kind of "valleys 
separated by ridges". This physical picture first suggested by Wannier for a particular class of reactions was discussed by Fano [36.37] in general case. If a system occupies some place on some ridge then its trajectory can either go down into some valley where a bound state of some fragments is created, or continue to propagate along the ridge. For the total fragmentation one should find a classical trajectory along a top of some ridge which leads from the region of small separation of fragments into the final state with infinite separation. It is clear that the lower is the above-threshold energy, the closer a trajectory should be to the top of the ridge.

Generally speaking there might exist several such ridges which lead to the final state with total fragmentation. In this work we study a particular ridge, which will be called the scaling configuration (SC). For all the systems considered up to now we have found that this configuration exists. More than that, for a given system there may exist several different SCs. A number of examples demonstrating this property is considered in Section D. Therefore one might suspect that SC is a general feature, though this latter statement is not verified so far. For two electrons in the field of an ion this ridge coincides with the Wannier ridge.

The basic idea is simple. As was said above one has to be sure that distances separating fragments monotonically increase with time. This condition is definitely satisfied if a trajectory describing $N$ particles which have masses $m_{j}(j=1,2, \cdots N)$ obeys the following conditions

$$
\vec{r}_{j}(t)=\phi(t) \vec{\rho}_{j}, \quad j=1,2, \cdots N,
$$

which are valid in the center of mass reference frame $\sum_{j} m_{j} \vec{r}_{j}(t)=0$. We shall refer to a trajectory satisfying Eq.(1) as SC. The time-independent vectors $\vec{\rho}_{j}$ describe the shape of the $\mathrm{SC}$, while the function $\phi(t)$ gives the overall scaling factor. We will see below that this function monotonically increases in time thus ensuring that all distances increase as well. Therefore this type of motion definitely results in total fragmentation avoiding traps into potential valleys. It is convenient to normalize the scaling function to unity for some initial 
moment of time $t_{0}$

$$
\phi\left(t_{0}\right)=1
$$

For this normalization the vectors $\vec{\rho}_{j}$ play a role of coordinates of the particles at this initial moment of time $\vec{r}_{j}\left(t_{0}\right)=\vec{\rho}_{j}$ in the center of mass reference frame

$$
\sum_{j} m_{j} \vec{\rho}_{j}=0
$$

Notice that in SC all degrees of freedom except the only one describing the overall scaling factor are frozen. In this sense the SC describes a quasiequilibrium of the system.

It is obvious that to satisfy (11) one should choose appropriately the initial coordinates $\vec{\rho}_{j}$. Let us formulate restrictions on them. Notice first of all that in the SC the accelerations of the particles are

$$
\frac{d^{2} \vec{r}_{j}(t)}{d t^{2}}=\frac{d^{2} \phi(t)}{d t^{2}} \vec{\rho}_{j}
$$

We presume purely Coulomb interaction, or consider Coulomb asymptote in more complicated cases, which is possible because the important distances are large $\left(r \sim r_{C}=1 / E\right)$ for low above-threshold energy. Therefore the potential energy of the system of $N$ fragments is

$$
U=\sum_{m>n} \frac{q_{m} q_{n}}{\left|\vec{r}_{m}-\vec{r}_{n}\right|}
$$

Here $q_{j}$ is a charge of a $j$-th fragment. The forces $\vec{F}_{j}$ for SC are time-scaled as

$$
\vec{F}_{j}(t)=-\frac{\partial U}{\partial \vec{r}_{j}}=\frac{1}{\phi(t)^{2}} \sum_{n \neq j} q_{j} q_{n} \frac{\vec{\rho}_{j n}}{\rho_{j n}^{3}},
$$

where $\vec{\rho}_{j n}=\vec{\rho}_{j}-\vec{\rho}_{n}$. Substituting (田),(可) in the Newton equation of motion one finds the following relation

$$
\frac{d^{2} \phi(t)}{d t^{2}} m_{j} \vec{\rho}_{j}=\frac{1}{\phi(t)^{2}} \sum_{n \neq j} q_{j} q_{n} \frac{\vec{\rho}_{i n}}{\rho_{i n}^{2}} .
$$

It is easy to see that it can be satisfied only if two conditions are fulfilled. Firstly, the scaling function should satisfy an equation 


$$
\frac{d^{2} \phi(t)}{d t^{2}}=-\frac{\alpha}{\phi(t)^{2}}
$$

where $\alpha>0$ is a time-independent constant which is discussed in detail below. One obviously recognizes in (8) the equation describing a one-dimensional motion of a particle with unite mass and unite charge in the attractive Coulomb field created by the charge $\alpha$. Secondly, the validity of (7) needs that the vectors $\vec{\rho}_{j}$ satisfy the following system of equations

$$
\alpha \vec{\rho}_{j}=\vec{a}_{j} \equiv-\frac{1}{m_{j}} \sum_{k \neq j} q_{j} q_{k} \frac{\vec{\rho}_{j k}}{\rho_{j k}^{3}},
$$

They state that accelerations of each fragment $\vec{a}_{j}$ is proportional to its coordinate vector at the initial moment of time. Eqs.(9) are shown to arise as conditions which are necessary for existence of SC. It is easy to see that they provide sufficient conditions as well. To verify this statement let us assume that we have a solution of (9). Then we can consider a trajectory with the following initial conditions. Firstly, we can choose initial coordinates as $\vec{r}_{j}\left(t_{0}\right)=\vec{\rho}_{j}$. Secondly, we can always choose initial velocities be proportional to coordinates

$$
\frac{d \vec{r}_{j}\left(t_{0}\right)}{d t}=\beta r_{j}\left(t_{0}\right)
$$

where $\beta$ is some positive constant which depends on the energy, $\beta \sim \sqrt{E}$. From (9) we find that accelerations at the initial moment of time are also proportional to coordinates

$$
\frac{d^{2} \vec{r}_{j}\left(t_{0}\right)}{d t^{2}}=\alpha \vec{r}_{j}\left(t_{0}\right)
$$

Thus for the considered trajectory both the velocities and accelerations linearly depend on coordinates at the initial moment of time. Combining this fact with the Newton equations of motion we conclude that the velocities (and accelerations) remain to be proportional to the coordinates for any moment of time

$$
\frac{d \vec{r}_{j}(t)}{d t}=\beta(t) \vec{r}_{j}(t)
$$

Here $\beta(t)$ is some positive function, $\beta\left(t_{0}\right)=\beta$. Integrating (12) we conclude that the time variation of distances does exhibit scaling condition (1), in which $\dot{\phi}(t)=\beta(t)$. 
This discussion shows that the SC (四) exists if and only if Eqs.(9) are satisfied. There are $N$ vector variables $\vec{\rho}_{j}, j=1,2, \cdots N$ and one scalar variable $\alpha$ in these equations. Obviously not all of them are independent because there are seven transformations which do not change the given SC. Three of them correspond to shifts of the SC center of mass. Three others describe rotations of the SC as a whole. One more transformation describes the overall scaling of SC

$$
\begin{aligned}
& \vec{\rho}_{j} \rightarrow \vec{\rho}_{j}^{\prime}=\lambda \vec{\rho}_{j}, \quad j=1,2, \cdots N \\
& \alpha \rightarrow \alpha^{\prime}=\lambda^{-3} \alpha
\end{aligned}
$$

with $\lambda>0$. According to Eq.(8) the scaling of $\alpha$ (14) should be accompanied by a corresponding scaling of $\phi(t)$, namely $\phi(t) \rightarrow \phi^{\prime}(t)=\lambda^{-1} \phi(t)$. Notice that the latter transformation can be interpreted as a shift of the initial moment of time

$$
t_{0} \rightarrow t_{0}^{\prime}
$$

where according to Eq.(2) $t_{0}^{\prime}$ should satisfy

$$
\phi^{\prime}\left(t_{0}^{\prime}\right)=\lambda^{-1} \phi\left(t_{0}^{\prime}\right)=1
$$

It is easy to see that Eqs.(9) remain invariant under the discussed above seven transformations, i.e. the shifts, rotations and scaling, allowing one to consider them as a set of $3 N-7$ equations for $3 N-7$ independent variables. When solving these equations it is convenient to treat $\alpha$ as a constant parameter which governs the overall scale and can be chosen arbitrary (for example $\alpha=1$ ).

At SC the system Hamiltonian

$$
H=\sum_{j=1}^{N} \frac{\vec{p}_{j}^{2}}{2 m_{j}}+U, \quad \vec{p}_{j}=m_{j} \frac{d \vec{r}_{j}}{d t}
$$

is reduced to

$$
H_{0}=\frac{1}{2} \mathcal{M}\left(\frac{d \phi}{d t}\right)^{2}-\frac{\mathcal{Q}_{0}}{\phi}
$$


where

$$
\begin{aligned}
\mathcal{M} & =\sum_{j=1}^{N} m_{j} \vec{\rho}_{j}^{2}, \\
\mathcal{Q}_{0} & =-\sum_{i>j} \frac{q_{i} q_{j}}{\left|\vec{\rho}_{i}-\vec{\rho}_{j}\right|} .
\end{aligned}
$$

Clearly the Hamiltonian (18) describes the one-dimensional motion of a particle with the mass $\mathcal{M}$ and unit charge in the attractive field of Coulomb center with the charge $-\mathcal{Q}_{0}$. The corresponding equation of motion is given by the considered previously Eq.(8) in which the constant $\alpha$ proves to be equal to

$$
\alpha=\frac{\mathcal{Q}_{0}}{\mathcal{M}}
$$

The interesting for us physical events take place if there is enough Coulomb attraction in the system. That is why we suppose that the effective Coulomb charge $\mathcal{Q}_{0}$ is attractive, $\mathcal{Q}_{0}>0$, resulting in positive value of $\alpha$.

Eqs.(19),(20),(21) show that arbitrary scaling of $\alpha$ can be compensated for by the corresponding scaling of coordinates $\rho_{j}$. This fact agrees with Eqs.(13),(14).

The scaling function $\phi(t)$ is defined by straightforward integration of (8)

$$
\frac{1}{2} \mathcal{M}\left(\frac{d \phi}{d t}\right)^{2}-\frac{\mathcal{Q}_{0}}{\phi}=E
$$

where $E$ is the system energy. Combined with the initial condition $\phi\left(t_{0}\right)=1$ this fixes the scaling function unambiguously.

It is important to emphasize that Eqs.(1), (9) present the idea of SC in an invariant form independent of the chosen coordinate frame. To see this more clearly let us introduce grand vectors in the $3 N$ dimensional configuration space. The grand vector $\mathbf{r}(t)=\left(\vec{r}_{1}(t), \cdots \vec{r}_{N}(t)\right)$ defines the time-dependent coordinates, the vector $\boldsymbol{\rho}=\left(\vec{\rho}_{1}, \cdots \vec{\rho}_{N}\right)$ gives the initial coordinates and $\mathbf{a}=\left(\vec{a}_{1}, \cdots \vec{a}_{N}\right)$ is the vector of accelerations at the initial moment of time. We employ bold type to distinguish such a vector from the conventional vector in space. Eqs.(1),(9) allow the following presentation 


$$
\begin{aligned}
\mathbf{r}(t) & =\phi(t) \boldsymbol{\rho}, \\
\alpha \boldsymbol{\rho} & =\mathbf{a} .
\end{aligned}
$$

Obviously, these relations between $3 N$-vectors do not depend on a reference frame. This shows that the scaling coordinate $\phi(t)$ is described in an invariant way.

It has been presumed by previous authors that some coordinate which describes fragmentation is to be singled out and the potential extremum point is to be found for the fixed value of this "break-up coordinate". The latter has been chosen in most cases as the system hyperradius [36] [37] [38] [39] defined as $R^{2}=\sum_{i=1}^{N} m_{i} r_{i}^{2}$. In the hyper-coordinate reference frame the potential energy

$$
V=\frac{C(\omega)}{R}
$$

is proportional to hypercharge $C(\omega)$ which depends on a set of hyperangles $\omega=$ $\left(\omega_{1}, \cdots \omega_{3 N-7}\right)$. It is easy to verify that definition of the $\mathrm{SC}$ (24) in the hyperspherical coordinates is reduced to

$$
\frac{\partial C(\omega)}{\partial \omega_{i}}=0
$$

which shows that a $\mathrm{SC}$ is a saddle-point of the hypercharge $C(\omega)$. The function $\phi(t)$ in hyperspherical coordinates is proportional to the hyperradius $\phi(t)=R / R_{0}$, where $R_{0}$ is the initial value of the hyperradius. The effective charge $\mathcal{Q}_{0}$ and the effective mass $\mathcal{M}$ can be expressed in terms of $R_{0}$ and the hypercharge $\mathcal{M}=R_{0}^{2}, \quad \mathcal{Q}_{0}=-C_{0} / R_{0}$ where $C_{0}$ is the hypercharge evaluated for SC. Description of the system in the hyper-coordinates has a long tradition and list of achievements, see for the example recent calculations of the three-electron atom in hyperspherical coordinates [40]. However, generally speaking, these coordinates do not possess fundamental advantages over other coordinate frames for the fragmentation problem.

Another well known reference frame provide Jacoby coordinates used in the approach developed by Feagin [41]. For the multiparticle fragmentation the choice of the "break-up coordinate" is not obvious and some special procedure was developed for its construction 
[29] [30] [19]. It becomes the more sophisticated problem the more complicated the system is.

In conclusion of this Section it should be stressed once more that our approach provides an invariant definition for the idea of SC which is given in (23), (24).

\section{SMALL DEVIATIONS FROM SCALING CONFIGURATION}

Assuming that the function $\phi(t)$ is defined as described in the preceding section, we switch from $\vec{r}_{j}$ to the new coordinates $\delta \vec{r}_{j}$

$$
\vec{r}_{j}=\phi(t) \vec{\rho}_{j}+\delta \vec{r}_{j}
$$

which have an obvious meaning of deviations from the SC. Presuming that these deviations are small, we write down linearized classical Newtonian equations for $\delta \vec{r}_{j}(t)$ as

$$
\begin{gathered}
m_{i} \frac{d^{2} \delta \vec{r}_{i}}{d t^{2}}=-\frac{1}{\phi(t)^{3}} \sum_{j=1}^{N} V_{i j} \delta \vec{r}_{j}, \\
V_{i j}=\frac{\partial^{2}}{\partial \vec{\rho}_{i} \partial \vec{\rho}_{j}} \sum_{m>n} \frac{q_{m} q_{n}}{\left|\vec{\rho}_{m}-\vec{\rho}_{n}\right|} .
\end{gathered}
$$

These equations of motion are generated by the time-dependent Hamiltonian function

$$
\delta H=\frac{1}{2} \sum_{j=1}^{N} \frac{\delta \vec{p}_{j}^{2}}{m_{j}}+\frac{1}{2 \phi(t)^{3}} \sum_{i, j=1}^{N} V_{i j} \delta \vec{r}_{i} \cdot \delta \vec{r}_{j}, \quad \delta \vec{p}_{j} \equiv m_{j} \frac{\delta \vec{r}_{j}}{d t} .
$$

It is convenient to introduce scaled deviations $\vec{\xi}_{j}$ and related momenta $\vec{\pi}_{j}$ as

$$
\vec{\xi}_{j}=\frac{1}{\phi(t)^{3 / 4}} \delta \vec{r}_{j}, \quad \vec{\pi}_{j}=\phi(t)^{3 / 4} \delta \vec{p}_{j}
$$

since this allows us to factor out the time dependence in the Hamiltonian:

$$
\delta H=\frac{1}{\phi(t)^{3 / 2}}\left[\frac{1}{2} \sum_{j=1}^{N} \frac{\vec{\pi}_{j}^{2}}{m_{j}}+\frac{1}{2} \sum_{i j} V_{i j} \vec{\xi}_{i} \vec{\xi}_{j}-\frac{3}{8} \sqrt{\phi} \frac{d \phi}{d t} \sum_{j=1}^{N}\left(\vec{\xi}_{j} \cdot \vec{\pi}_{j}+\vec{\pi}_{j} \cdot \vec{\xi}_{j}\right)\right] .
$$

The derivation of this formula could be traced via a quantum mechanical analogue of the problem (which for many readers nowadays is more convenient than the pure classical consideration). In quantum mechanics the transformation rules for momenta and the Hamiltonian follow respectively from the formulae for the partial derivatives 


$$
\frac{\partial}{\partial \vec{r}_{j}}=\phi(t)^{-3 / 4} \frac{\partial}{\partial \vec{\xi}_{j}}, \quad\left(\frac{\partial}{\partial t}\right)_{\delta \vec{r}_{j}}=\left(\frac{\partial}{\partial t}\right)_{\vec{\xi}_{j}}+\frac{3}{4 \phi(t)^{1 / 4}} \frac{d \phi}{d t} \sum_{j=1}^{N} \vec{\xi}_{j} \cdot \frac{\partial}{\partial \delta \vec{r}_{j}} .
$$

In (32) we use a symmetrized representation which should be employed in the quantum version of the formulae (the latter also implies a corresponding gauge transformation for the wave function).

From (22) one obtains

$$
\sqrt{\phi} \frac{d \phi}{d t}=\sqrt{\frac{2\left(E \phi(t)+\mathcal{Q}_{0}\right)}{\mathcal{M}}}
$$

which becomes time-independent for $E=0$. In this case the time-dependence is exactly factored out in the Hamiltonian (32) justifying the choice of the scaling (31). This implies that the original non-stationary problem becomes stationary provided one replaces time $t$ by an effective time $\tau$. A relation between $t$ and $\tau$ in differential form is

$$
d \tau=\phi(t)^{-3 / 2} d t
$$

For some applications it is necessary to keep the energy dependence of the trajectory. For these cases a convenient technique has been developed recently by Kuchiev [17]. We have applied it to the case considered and verified that it results in the same threshold indexes as the ones obtained below by the stationary approach.

The Hamiltonian describing propagation in the effective time (35) reads

$$
\begin{aligned}
& \delta H_{\tau}=\frac{1}{2} \sum_{j=1}^{N} \frac{\vec{\pi}_{j}^{2}}{m_{j}}+\frac{1}{2} \sum_{i j} V_{i j} \vec{\xi}_{i} \vec{\xi}_{j}+\frac{a}{2} \sum_{j=1}^{N}\left(\vec{\xi}_{j} \cdot \vec{\pi}_{j}+\vec{\pi}_{j} \cdot \vec{\xi}_{j}\right), \\
& a=-\frac{3}{4} \sqrt{\phi} \frac{d \phi}{d t} .
\end{aligned}
$$

The Hamiltonian $\delta H_{\tau}$ (36) is quadratic in coordinates and momenta thus describing a set of harmonic oscillators or inverted oscillators. This shows that our goal is to describe the behavior of the system in terms of these oscillators and inverted oscillators. Before proceeding we modify our notation. The set of components of the displacements vectors $\delta \vec{r}_{j}$ $(j=1,2, \ldots N)$ comprise $3 N$-dimensional grand vector $\delta \mathbf{r}$. In this formulation, for instance, $V_{i j}$ corresponds to grand $3 N \times 3 N$ square matrix denoted below as $\mathbf{V}$. We introduce also 
$3 N \times 3 N$ unit matrix $\mathbf{I}$ and the diagonal matrix $\mathbf{K}$ of the same size with diagonal elements corresponding to inverse mass $1 / m_{j}$ of each particle.

This notation takes into account an obvious fact that the total number of all modes coincides with the number of degrees of freedom in the system $(k=1,2, \ldots 3 N)$. There are however seven particular degrees of freedom: translations, rotations and the scaling transformation. They do not change the shape of a SC and do not describe a deviation from a SC. These degrees of freedom may be called the collective modes. They obviously should be considered separately from the oscillating modes which describe deviations from the SC. In order to distinguish the collective modes one can use the following interesting property. All collective degrees of freedom are described by the eigenvectors of the grand matrix $\mathbf{K V}$ with particular eigenvalues. Firstly, the three modes which correspond to the system translations in space have obviously zero eigenvalues. Secondly, the modes corresponding to rotations of the system in space have eigenvalues equal to $\mathcal{Q}_{0} / \mathcal{M}$, as shown in Appendix. There are three such modes in general case, while for a linear SC there are only two modes. Thirdly, the mode corresponding to the scaling transformation Eqs.(113),(14) has an eigenvalue $-2 \mathcal{Q}_{0} / \mathcal{M}$, as also shown in Appendix. Using these eigenvalues one can separate the collective modes either from the very beginning, or at the end of calculations.

There is another useful for applications way to separate the collective modes. For translations and rotations the separation can be fulfilled by conventional methods choosing appropriately the coordinates, as is demonstrated in a number of examples below. Separation of the scaling mode can be achieved with the help of the operator of projection on this mode $\mathbf{P}$ and the complementary projection operator $\mathbf{Q}=\mathbf{I}-\mathbf{P}$. The operator $\mathbf{P}$ is readily constructed from the unit vectors $\vec{n}_{j}=\vec{\rho}_{j} / \rho_{j}$ which define the shape of SC:

$$
P_{i j}=\vec{n}_{i} \cdot \vec{n}_{j} .
$$

Thus all seven collective modes can be easily identifies and separated using any of the two techniques described above.

Some modes in the $3 N-7$ subspace orthogonal to the collective modes are stable and 
describe small oscillations around SC; the related oscillating frequencies $\omega_{k}$ are real. The object of our major interest is unstable modes with imaginary oscillating frequencies. It is shown below that unstable modes exist for any SC. It is convenient to introduce for unstable modes a parameter $\alpha_{k}=i \omega_{k}\left(\operatorname{Re} \alpha_{k}>0\right)$. In order to find the oscillating frequencies one can presume a harmonic time-dependence of the coordinates $\xi$ and momenta $\pi$

$$
\boldsymbol{\xi}=\exp (i \omega t) \boldsymbol{\Xi}, \quad \boldsymbol{\pi}=\exp (i \omega t) \boldsymbol{\Pi}
$$

where $\boldsymbol{\Xi}$ and $\boldsymbol{\Pi}$ are time-independent grand vectors. The Hamiltonian equations of motion give

$$
i \omega \Xi=\mathbf{K} \boldsymbol{\Pi}+a \boldsymbol{\Xi}, \quad i \boldsymbol{\Pi}=-\mathbf{V} \boldsymbol{\Xi}-a \boldsymbol{\Pi},
$$

where $a(37)$ is a scalar coefficient. The latter equation could be written also as

$$
i \omega\left(\begin{array}{c}
\Xi \\
\Pi
\end{array}\right)=\left(\begin{array}{cc}
a & \mathbf{K} \\
-\mathbf{V} & -a
\end{array}\right)\left(\begin{array}{c}
\boldsymbol{\Xi} \\
\boldsymbol{\Pi}
\end{array}\right) .
$$

Excluding the grand vector $\boldsymbol{\Pi}$ one comes to the eigenvalue problem for the square of frequency $\omega^{2}$

$$
\left(\omega^{2}+a^{2}\right) \boldsymbol{\Xi}=\mathbf{K} \mathbf{V} \boldsymbol{\Xi}
$$

or, in the symmetrized form

$$
\left(\omega^{2}+a^{2}\right) \tilde{\boldsymbol{\Xi}}=\mathbf{K}^{1 / 2} \mathbf{V} \mathbf{K}^{1 / 2} \tilde{\boldsymbol{\Xi}}, \quad \mathbf{K}^{1 / 2} \tilde{\boldsymbol{\Xi}}=\boldsymbol{\Xi}
$$

Denoting a set of eigenvalues of the matrix $\mathbf{K} \mathbf{V}$ as $v_{k}, k=1,2, \cdots, 3 N$, we obtain

$$
\begin{gathered}
\omega_{k}^{2}=v_{k}-a^{2}, \\
\alpha_{k}=\sqrt{a^{2}-v_{k}} .
\end{gathered}
$$

This formula shows how the oscillation frequencies depend on the eigenvalues of the matrix K V. 
Let us verify now that a $\mathrm{SC}$ is always unstable. With this purpose let us show that the matrix K V always possesses negative eigenvalues which describe instability. Consider the trace of the grand matrix $\mathbf{V}$

$$
\operatorname{Tr} \mathbf{V}=\sum_{j} \frac{\partial^{2}}{\partial \vec{\rho}_{j} \partial \vec{\rho}_{j}} \sum_{m>n} \frac{q_{m} q_{n}}{\left|\vec{\rho}_{m}-\vec{\rho}_{n}\right|}=\sum_{j} \triangle_{\vec{\rho}_{j}} \sum_{m>n} \frac{q_{m} q_{n}}{\left|\vec{\rho}_{m}-\vec{\rho}_{n}\right|}=0
$$

which vanishes since the Coulomb potential satisfies the Laplace equation

$$
\triangle_{\vec{\rho}_{j}} \frac{1}{\left|\vec{\rho}_{j}-\vec{\rho}_{n}\right|}=0 \quad \vec{\rho}_{j} \neq \vec{\rho}_{n}
$$

It is easy to see also that (46) results in $\operatorname{Tr}(\mathbf{K ~ V})=0$ which means that

$$
\operatorname{Tr}(\mathbf{K ~ V})=\sum_{k} v_{k}=0
$$

We see that the spectrum of the matrix $\mathbf{K ~ V}$ always contains both positive and negative eigenvalues. This fact in itself is not sufficient to make a statement about instability because the trace (47) includes contribution from collective modes which do not change a shape of the SC. However, it is easy to exclude collective modes. Remember that the eigenvalues corresponding to translations are zero, rotations give eigenvalues $\mathcal{Q} / \mathcal{M}$, while the scaling transformation provides the eigenvalue $-2 \mathcal{Q} / \mathcal{M}$, see Appendix. The sum of eigenvalues of collective modes is

$$
\sum_{\text {collective modes }} v_{k}=\left\{\begin{array}{rc}
\mathcal{Q} / \mathcal{M} & \text { in general case } \\
0 & \text { for linear SC. }
\end{array}\right.
$$

Subtracting this result from (47) we find the trace of the matrix $\mathbf{K ~ V}$ in the subspace orthogonal to the collective modes

$$
\operatorname{Tr}(\mathbf{K ~ V})_{\text {orth }}=\sum_{\text {orthogonal }} v_{k}=\left\{\begin{array}{rr}
-\mathcal{Q} / \mathcal{M} & \text { in general case } \\
0 & \text { for linear SC. }
\end{array}\right.
$$

Since this trace is non-positive, we conclude that the matrix $\mathbf{K} \mathbf{V}$ inevitably possesses negative eigenvalues which describe deviations from the SC. This shows that any SC is unstable. This property is closely related to the fact that harmonic functions, i.e. those which satisfy 
the Laplace equation, cannot have maxima or minima. Remember also the Earnshow theorem well known in electrostatics: stable equilibrium is impossible for systems where the Coulomb forces are operative. Although SCs describe expanding non-static configurations, conclusion about inevitable instability remains valid in this case as well. This fact can be interpreted as a dynamic analogue of the Earnshow theorem.

\section{QUANTIZATION OF DEVIATIONS FROM SCALING CONFIGURATION AND THRESHOLD INDICES}

Previous section reduces description of small deviations from SC to the set of coupled harmonic oscillators which could be quantized straightforwardly. This procedure provides the 'energy' levels

$$
\epsilon_{k n_{k}}=\omega_{k}\left(n_{k}+\frac{1}{2}\right)
$$

Here the first subscript $k=1,2, \cdots, 3 N-7$ indicates the mode, and $n_{k}=0,1, \cdots$ shows a

number of quanta in this mode. For a given set of the quantum numbers $\left\{n_{k}\right\}$ the system wave function is given by

$$
\Psi_{\left\{n_{k}\right\}} \sim \exp \left(-\sum_{k} i \int_{\tau_{0}}^{\tau} \epsilon_{k n_{k}} d \tau\right)=\exp \left(-\sum_{k} i \int_{t_{0}}^{t} \frac{\epsilon_{k n_{k}}}{\phi(t)^{3 / 2}} d t\right)
$$

where we omit the common time-dependent phase factor. The wave function is prepared at some initial moment $t_{0}$ by preceding strong interaction of all fragments. In the Wanniertype approach it is presumed that these processes depend smoothly on the energy E. Hence they do not influence the form of threshold law and thus could be effectively excluded from consideration; it is sufficient to consider only $t>t_{0}$ domain.

For unstable modes the 'energies' $\epsilon_{k n_{k}}$ are complex-valued which leads to the loss of probability in the expanding SC. This should be interpreted [34] [35] as sliding from the potential saddle in multidimensional configuration space that eventually leads to formation of bound states of two (or more) fragments. Such an outcome implies that the related part 
of probability is lost for the process of complete system fragmentation which is an object of our study. Cross section of the latter is proportional to the survival probability

$$
\left.P_{\left\{n_{k}\right\}} \equiv\left|\Psi_{\left\{n_{k}\right\}}\right|_{t \rightarrow \infty}\right|^{2}=\exp \left(-\sqrt{2 \mathcal{M}} \sum_{k} \int_{\phi\left(t_{0}\right)}^{\infty} \frac{\alpha_{k n_{k}}}{\phi \sqrt{E \phi+\mathcal{Q}_{0}}} d \phi\left(n_{k}+\frac{1}{2}\right)\right)
$$

where summation over $k$ runs over all unstable modes. Note that the original quantum problem is stationary. The time $t$ in (52) plays a role of an effective variable which describes scaling of the system in accordance with (22). Small deviations from SC are described quantum mechanically. Our treatment generalizes to multimode case the scheme developed by Kazansky and Ostrovsky [4] for the two-electron escape (see also Ref. [12]; some of ideas used were elaborated also by Watanabe [2]). Note that the cited paper [4] provides also a description of deviations from pure power threshold law, but we do not pursue this point here.

For our objectives it is sufficient to note that (52) has a form of a product of contributions coming from each individual mode, hence the threshold law of interest is

$$
\begin{array}{r}
\sigma \sim P_{\left\{n_{k}\right\}} \sim E^{\mu} \\
\mu \equiv \sum_{k} \mu_{k n_{k}}
\end{array}
$$

The partial threshold indices $\mu_{k n_{k}}$ stem from the 'eigenfrequencies' of unstable modes being related to the negative eigenvalues $v_{k}<0$ of the $\mathbf{K ~ V}$ matrix

$$
\mu_{k n_{k}}=2\left[\sqrt{-\frac{\mathcal{M}}{2 \mathcal{Q}_{0}} v_{k}+\frac{9}{16}}-\frac{1}{4}\right]\left(n_{k}+\frac{1}{2}\right) .
$$

Small positive values of $v_{k}$ formally could also lead to real $\mu_{k n_{k}}$, but have to be discarded. Obviously, if some (imaginary) 'eigenfrequencies' are $N_{k}$-fold degenerate, the related contributions appear $N_{k}$ times in the sum (54). In principle the wave function is a superposition of terms corresponding to various sets of quantum numbers $\left\{n_{k}\right\}$, since all of them are populated by the processes in the inner interaction domain. Clearly, the threshold law is defined by the least possible values of $n_{k}$ [42] which are equal to zero unless the symmetry considerations forbid this choice, as exemplified in the next paragraph. If the initial SC is scaled 
by the factor $\lambda$, see Eqs.(13), (14), then $\mathbf{K ~ V ~} \sim \lambda^{-3}, \mathcal{M} \sim \lambda^{2}, \mathcal{Q}_{0} \sim \lambda^{-1}$, but the threshold indices $\mu_{k n_{k}}$, as anticipated, remain scale-independent. Note also that the threshold index is invariant under simultaneous scaling of all charges or all masses in the system.

In the original Wannier problem two electrons escape from infinitely heavy atomic core with the charge $Z$. The configuration found by Wannier [1] gives the simplest example of SC in which the electrons reside at equal distances $\rho$ and in opposite directions from the core. The motion is unstable with respect to the stretching mode which is separated from the (stable) bending mode. Thus it is sufficient for our purposes to consider motion of electrons along the line passing through the core. This motion is described by two coordinates and the matrix $\mathbf{V}$ takes the form

$$
\mathbf{V}=\frac{1}{\rho^{3}}\left(\begin{array}{cc}
-2 Z+\frac{1}{4} & -\frac{1}{4} \\
-\frac{1}{4} & -2 Z+\frac{1}{4}
\end{array}\right)
$$

and $\mathcal{Q}_{0}=\left(2 Z-\frac{1}{2}\right) \rho^{-1}, \mathcal{M}=2 \rho^{2}$ (we use atomic system of units, $\mathbf{K}=\mathbf{I}$ ). The eigenvalues of $\mathbf{V}$ are $v_{1}=-2 Z / \rho^{3}$ and $v_{2}=\left(-2 Z+\frac{1}{2}\right) / \rho^{3}$. The eigenvalue $v_{2}$ is seen to coincide with $-2 \mathcal{Q} / \mathcal{M}$. Hence it corresponds to $\mathrm{SC}$ expansion (see Appendix) and should be discarded. The eigenvalue $v_{1}$ upon substitution into (55) reproduces the well known result

$$
\mu_{1 n_{1}}=\frac{1}{2}\left[\sqrt{\frac{100 Z-9}{4 Z-1}}-1\right]\left(n_{1}+\frac{1}{2}\right) .
$$

The choice $n_{1}=0$ provides the famous Wannier law valid for ${ }^{1} S$ symmetry of the final two-electron continuum state, whereas $n_{1}=1$ corresponds to the threshold law for ${ }^{3} S^{e}$ (and $\left.{ }^{3} P^{e}\right)$ symmetry [43].

Feagin and Filipczyk [29] and Poelstra el al [30] put forward another formula for the threshold index in the multimode case. According to it the Wannier index is $(N-2)$ times larger than (54). The factor $(N-2)$ is described as a "phase space factor for $(N-1)$ outgoing particles" being justified by the reference to the earlier paper by Feagin [41]. We were unable to find the derivation of such a factor in the cited paper; anyway it deals only with the conventional $N=3$ case where the factor $(N-2)$ is insignificant. Our treatment 
provides purely dynamic approximation for the wave function and does not leave any room for the statistical arguments. The other aspects of relation between dynamic and statistical threshold laws are discussed in Section VI.

\section{PARTICULAR SYSTEMS}

In practical applications of our scheme the less obvious part corresponds to finding SCs. Numerical solution of the set of non-linear equations (9) could be cumbersome and implies reasonable initial guess. The question whether all the solutions are found is even more difficult. In reality one has to appeal to intuitive reasoning and to limit search to some symmetrical configuration. This allows one to effectively reduce the number of equations (9) to be considered. Since the initial step of finding SC in most cases could not be done in closed form, we do not pursue the goal of obtaining analytical formulae, but resort to numerical calculations which are performed using the Mathematica [44] program. We find it easier to avoid preliminary separation of rotational and translational coordinates, since they could be easily distinguished in the eigensystem of the complete matrix $\mathbf{K ~ V}$. Moreover, the known eigenvalues of this matrix corresponding to rotations (see Appendix) provide a good test for consistency of calculations.

The systems practically accessible nowadays in atomic physics are not very diverse, consisting of several electrons and positrons in the field of heavy (positively charged) atomic

core. Since three-particle systems ( such as $\mathrm{A}^{+Z}+2 e^{-}$or $\mathrm{A}^{+Z}+e^{-}+e^{+}$) are already studied in great detail [四] [38] [39] 45] [46] [47] [33] (see also references in the Introduction), we start from the four-particle systems. We do not impose any symmetry constraints on the system state thus presuming that $n_{k}=0$ for all modes contributing $\mu$ (54).

\section{A. Three-electron escape from the charged core}

The system $\mathrm{A}^{+Z}+3 e$ was thoroughly investigated by Klar and Schlecht [22] and Grujić [24]. They considered a configuration of electrons forming an equilateral triangle with in- 
finitely massive core in the center, which is obviously a SC. The out-of-plane motion is separated. It corresponds to stable modes and does not affect the threshold law. The inplane motion is described by six coordinates of electrons, or by four 'oscillatory' modes plus uniform expansion of SC and its rotation. The eigenfrequencies obtained by us, as well as in the cited papers, are pairwise degenerate due to SC symmetry. One pair corresponds to stable motion and the other pair to unstable motion. The latter pair produces two equal terms in the sum (54). Klar and Schlecht 222 and Grujić 24 succeeded in deriving analytical expressions for the Wannier index [48. In this paper we do not pursue analytical formulations but check that our numerical results coincide with those cited by Grujić, namely $\mu=2.82624$ for $Z=1, \mu=2.27043$ for $Z=2, \mu=2.16196$ for $Z=3$, etc. The experiment for electron impact double ionization of atoms $(Z=3)$ seem to agree with the threshold law 28 .

The two pairs of modes discussed above are already well known. Combined with rotation and scaling expansion they represent a complete set of six in-plane coordinates. Since the number of modes is a physical parameter which is independent on the theoretical technique used, we do not see any possibility to obtain some additional unstable modes which would lead to another Wannier index and thus to the complementary threshold law as announced by Feagin and Filipczyk [29] (in fact our conclusion could be drawn from the paper by Grujić [24] who used the plain Cartesian coordinates whereas less transparent treatment by Klar and Schlecht [22] is based on hyperspherical coordinates). Since no details of analysis by Feagin and Filipczyk [29] were ever published, more detailed discussion of this issue is not possible.

\section{B. $2 e^{-}+e^{+}$escape from the charged core}

The plausible symmetric SCs for the system $\mathrm{A}^{+Z}+2 e^{-}+e^{+}$were considered by Poelstra et al [30] (note that the calculations in this paper were carried out only for $Z=1$ ). They comprise two different linear arrangements and one plain configuration [49]. All these 
configurations belong to SC and therefore can be easily handled by the developed above technique. We consider below these SCs successively.

\section{Linear configuration $L_{a}$}

Let the frame origin is placed into infinitely massive core having the charge $Z$. The coordinates of two electrons and positron are respectively $x_{1}, x_{2}, x_{3}$; all of them are positive. It is convenient to introduce two dimensionless parameters $x=r_{1} / r_{3}$ and $y=r_{2} / r_{3}(0<$ $x<1<y$ ) which have to satisfy the system of equations obtained from (9)

$$
\begin{gathered}
\frac{m_{3}}{m_{1}} \frac{Z / x^{2}-1 /(1-x)^{2}+1 /(y-x)^{2}}{-Z+1 /(1-x)^{2}-1 /(1-y)^{2}}=x \\
\frac{m_{3}}{m_{2}} \frac{Z / y^{2}+1 /(1-y)^{2}-1 /(y-x)^{2}}{-Z+1 /(1-x)^{2}-1 /(1-y)^{2}}=y
\end{gathered}
$$

(equations are presented for more general case when all light particles have different masses $m_{i}$, while the core remains infinitely heavy).

\section{Linear configuration $L_{b}$}

A distinction from the previous case is that the coordinate of one of the electrons is negative $\left(x_{2}<0\right)$. The system of equations defining $\mathrm{SC}$ is somewhat different $(y<0<x<$ $1)$ :

$$
\begin{gathered}
\frac{m_{3}}{m_{1}} \frac{Z / x^{2}-1 /(1-x)^{2}-1 /(y-x)^{2}}{-Z+1 /(1-x)^{2}+1 /(1-y)^{2}}=x \\
\frac{m_{3}}{m_{2}} \frac{-Z / y^{2}-1 /(1-y)^{2}+1 /(y-x)^{2}}{-Z+1 /(1-x)^{2}+1 /(1-y)^{2}}=y
\end{gathered}
$$

For both linear configurations the bending modes are stable. There are two stretching modes for each configuration, both being unstable. The results of our calculations are summarized in table 1 . For $Z=1$ parameters $x, y$ and partial threshold indices $\mu_{1}$ and $\mu_{2}$ coincide with those obtained by Poelstra et al [30]; our threshold indices $\mu$ are less by a factor of 2, as

discussed at the end of Section $[\mathrm{IV}$. Notice a non-trivial behaviour of the parameters with $Z$ : 
for instance, in SC $L_{a}, x$ and $\mu_{1}$ increase with $Z$, whereas $y$ and $\mu_{2}$ decrease. The threshold index $\mu$ increases with $Z$ which is opposite to the well known behaviour for the simplest system $\left(\mathrm{A}^{Z}+2 e\right)$ and for $3 e$ escape where $\mu$ diminishes as $Z$ grows (see more discussion in the Section $\nabla \mathrm{I})$.

\section{Plane configuration $P$}

The symmetric plane configuration is conveniently characterized by two angles $\alpha$ and $\beta$ : $\alpha$ is an angle between two lines which join the ion with the positron and one of the electrons, while $\beta$ is an angle between two lines which join the positron with the ion and with one of the electrons. In the considered plane configuration the two electrons are located symmetrically, which means that their locations mirrow each other under reflection in an axes which joins the ion and the positron. This makes the angles $\alpha$ and $\beta$ be identical for both electrons. From (9) we deduce the system of equations

$$
\begin{array}{r}
m_{+} \sin ^{3} \gamma\left(Z \sin ^{2} \alpha-\frac{1}{4} \sin \alpha+\sin ^{2} \beta \cos \gamma\right)= \\
=m_{-} \sin ^{3} \beta\left(2 \cos \beta \sin ^{2} \gamma-Z \sin ^{2} \alpha\right), \\
\sin ^{2} \beta \sin \gamma=\frac{1}{4} \cos \alpha \quad(\gamma=\pi-\alpha-\beta),
\end{array}
$$

where the masses of light particles with negative $\left(m_{-}\right)$and positive $\left(m_{+}\right)$charges generally could be different. The results of calculations are presented in table 1 . For $Z=1$ the angles $\alpha$ and $\beta$ coincide with these extracted from the paper by Poelstra et al [30]. However, the difference between the threshold indices is drastic. Poelstra et al had found a single unstable mode which corresponds to our partial Wannier index $\mu_{2}$. Our calculations give two unstable modes, similarly to the case of $3 e$ escape (in the latter case the modes were degenerate due to a symmetry which is absent for the system under consideration). The reason of this disagreement remains unclear.

The plane SC $P$ governs the threshold behaviour, although it provides the threshold index $\mu$ only slightly less than the linear configuration $L_{b}$. 


\section{Four-electron escape from the charged core}

Basing on the symmetry considerations we analyze three configurations: linear, plane and 3D SC. It could be shown rigorously that for symmetric linear arrangement SC does not exist for all values of $Z$, i.e. Eqs.(9) have no solution.

\section{Plane configuration $P$}

In the plane configuration the electrons are located in the apexes of a square; the core lies in its center. The out-of-plane motion is separated and corresponds to stable modes. For in-plane motion in general case we find one non-degenerate and one doubly-degenerate unstable modes (table 2). For the particular case $Z=1$ an additional non-degenerate mode becomes unstable.

\section{3D configuration $V$}

$\mathrm{SC}$ describes the electrons located at the apexes of tetrahedron. We find a single triplydegenerate mode (table 2). Interestingly, the threshold index $\mu$ proves to be quite close for plane and 3D configurations, although 3D SC provides somewhat lower value of $\mu$ and thus governs the threshold behaviour. As $Z$ increases, the relative importance of electron-electron interaction decreases and $\mu$ approaches the value $\mu=3$ which corresponds to non-interacting electrons.

The smallest practically attainable value of the charge seems to be $Z=2$. It could be realized via triple ionization of negative ion by electron impact. However, theoretically the case $Z=1$ proves be very interesting due to unusual properties. In this case the threshold index becomes much larger than in other cases, particularly for the plane SC. This is due to a small value of the 'charge' $\mathcal{Q}_{0}$ in this case. Another interesting feature is an appearance of an additional unstable mode in the plane SC. An analysis of the eigenvector $\boldsymbol{\Xi}$ shows that 
it corresponds to the out-of-plane motion. Namely, a pair of electrons lying on a diagonal of the square shifts upwards, whereas another pair shifts downwards.

The tetrahedric configuration was considered earlier by Grujić [26] who obtained approximate analytical expressions for the threshold indexes. The partial threshold indexes obtained by him reveals only an approximate degeneracy. The numerical results for $\mu$ are in reasonable agreement with our data.

\section{D. $3 e^{-}+e^{+}$escape from the charged core}

We failed to find a symmetrical plane SC for this system.

\section{Linear configuration $L$}

Linear SC corresponds to alternating positive and negative charges. Let us locate the origin at the heavy ion and call by $x_{1}>0, x_{2}<0, x_{3}>x_{1}>0$ locations of three electrons, and by $x_{4}, x_{1}<x_{4}<x_{3}$ location of the positron. Then the considered configuration can be characterized by three parameters: $x=x_{1} / x_{4}, y=x_{2} / x_{4}, z=x_{3} / x_{4}((y<0<x<1<z)$. They have to satisfy a set of equations which follow from (9)

$$
\begin{gathered}
-\frac{Z}{x^{2}}+\frac{1}{(x-y)^{2}}+\frac{1}{(1-x)^{2}}-\frac{1}{(z-x)^{2}}=x\left[Z-\frac{1}{(1-x)^{2}}+\frac{1}{(z-1)^{2}}-\frac{1}{(1-y)^{2}}\right] \\
\frac{Z}{y^{2}}-\frac{1}{(x-y)^{2}}+\frac{1}{(1-y)^{2}}-\frac{1}{(z-y)^{2}}=y\left[Z-\frac{1}{(1-x)^{2}}+\frac{1}{(z-1)^{2}}-\frac{1}{(1-y)^{2}}\right] \\
-\frac{Z}{z^{2}}+\frac{1}{(z-x)^{2}}+\frac{1}{(z-y)^{2}}-\frac{1}{(z-1)^{2}}=z\left[Z-\frac{1}{(1-x)^{2}}+\frac{1}{(z-1)^{2}}-\frac{1}{(1-y)^{2}}\right]
\end{gathered}
$$

The parameters of SC and the Wannier indices are shown in table 3.

\section{3D configuration $V$}

The symmetrical 3D configuration arises when the three electrons form the equilateral triangle while the ion and the positron are located up and down the plane of the triangle 
on the perpendicular to the triangle plane which crosses its center. This configuration is characterized by two angles $\alpha$ and $\beta$ defined similar to the case considered in Section B3. Namely, $\alpha$ is an angle between two lines which join the ion with the positron and with one of the electrons, while $\beta$ is an angle between two lines which join the positron with the ion and with one of the electrons. These angle are difined by equations similar to (59):

$$
\begin{array}{r}
m_{+} \sin ^{3} \gamma\left(Z \sin ^{2} \alpha-\frac{1}{\sqrt{3}} \sin \alpha+\sin ^{2} \beta \cos \gamma\right)= \\
=m_{-} \sin ^{3} \beta\left(3 \cos \beta \sin ^{2} \gamma-Z \sin ^{2} \alpha\right), \\
\sin ^{2} \beta \sin \gamma=\frac{1}{\sqrt{3}} \cos \alpha \quad(\gamma=\pi-\alpha-\beta) .
\end{array}
$$

We have found two doubly-degenerate and one non-degenerate unstable mode as shown in table 3. The threshold law is governed by 3D SC $V$. Note that the threshold index grows with $Z$.

\section{E. Five-electron escape from the charged core}

\section{Plane configuration $P$}

In the plane $\mathrm{SC}$ the electrons are located in the apexes of a equilateral pentagon; the

core lies in the same plane. In the in-plane motion we have found two doubly degenerate unstable modes (table 4 ). For $Z=2$ an additional pair of unstable modes appears.

\section{3D configuration $V$}

Here three electrons lie in the apexes of equilateral triangle with the core in its center. On the perpendicular to this plane, above the plane and below it, another pair of electrons is located symmetrically. The SC can be characterized by the angle $\alpha$ between the line which joins out-of-plane electron with the core and the line which joins it with in-plane electron. The angle is defined by the equation 


$$
\frac{1}{\sqrt{3}}+2 \sin ^{3} \alpha-Z=\tan \alpha\left(3 \sin ^{2} \alpha \cos \alpha+\frac{1}{4} \tan ^{2} \alpha-Z \tan ^{2} \alpha\right) .
$$

Quite unexpectedly, $\alpha$ proves to be very close to $45^{\circ}$, exhibiting weak dependence on the core charge $Z$ (table 4 ). This means that in-plane and out-of-plane electrons are located at

almost the same distance from the core. The 3D SC generates somewhat lower values of $\mu$ than the plane SC thus governing the threshold behaviour. However, the difference is quite small. This feature is common to that found above for the four-electron case.

3D configuration for five-electron system was considered previously by Dmitriević et al [27. However, the equation derived for SC angle $\alpha$ differs from (62).

\section{F. Fragmentation in two pairs of identical particles with opposite charges}

In this subsection we consider fragmentation into the final state $2 X_{m}^{+Z}+2 e$, where $X_{m}^{+Z}$ is a positively charged particle with charge $Z$ and mass $m$ (all results below hold if the electrons are replaced by any other charged particles; then $Z$ and $m$ have the meaning of ratio of charges and masses respectively). In the applications considered above the zero eigenvalues of the matrix $\mathbf{V}$ do not emerge due to the presence of infinitely massive core. In the $\left(2 X_{m}^{+Z}+2 e\right)$ system such modes are present. Another distinction is that for equal masses of leptons in previous applications we have always had $\mathbf{K}=\mathbf{I}$ and $v_{k}$ have been the eigenvalues of the $\mathbf{V}$ matrix. Now we have to diagonalize the complete matrix $\mathbf{K ~ V}$. Both these features do not create substantial difficulties.

From the symmetry considerations it is clear that a shape of the $\mathrm{SC}$ is a rhombus with the angle $2 \alpha$ at the apexes where the particles $X_{m}^{Z}$ are situated. The single SC parameter $\alpha$ is defined by the equation

$$
8 Z-\frac{Z}{\cos ^{3} \alpha}=m\left(8 Z-\frac{1}{\sin ^{3} \alpha}\right)
$$

which follows from (9). Several examples are shown in table 5. The simplest practical realization is the complete fragmentation of $\mathrm{H}_{2}$ molecule by photons where $\alpha$ is close to $30^{\circ}$ in agreement with Feagin and Filipczyk [29] and the threshold index proves to be huge. 
Apparently this threshold behaviour could not be observed in experiments [50]. Another feasible realization with moderate Wannier index is ionization of negative positronium ion by positron impact $(Z=1, m=1)$.

We fail to find the linear configuration discussed by Stevens and Feagin [31].

\section{DISCUSSION AND CONCLUSION}

This paper formulates the idea of the SC. Defined by (四), the SC is shown to arise when a nonlinear set of Eqs.(91) is satisfied. Propagation of the system in the vicinity of $\mathrm{SC}$ configuration governs the threshold law which is found in Eqs.(53),(54) and (55). These results permit direct practical calculations of the threshold index $\mu$ for any system.

In many cases the threshold laws in quantum mechanics can be deduced from general considerations without dynamical treatment. For instance, the break-up cross section with $N$ fragments in the final state and a short range interaction between them could be estimated from simple phase-space volume (i.e. statistical) arguments as

$$
\sigma_{\mathrm{s}} \sim E^{\frac{3}{2}(N-1)-1} .
$$

If one presumes that all fragments ("electrons") are attracted by Coulomb forces to one fragment ("core"), but the interaction between the "electrons" is negligible, then the phase space arguments could be easily modified to give

$$
\sigma_{\mathrm{C}} \sim E^{N-2}
$$

In case of repulsive Coulomb interaction with the "core" (but still without other interfragment interactions) the cross section at the threshold becomes exponentially small, as obtained, for example, by Geltman [51] in his calculations for atom ionization by positron impact with all correlation neglected. The threshold behaviour changes to $\sim E^{3 / 2}[52$ if one employs the so called $3 \mathrm{C}$ wave functions for the final continuum state. However, these functions do not ensure proper description in the near-threshold domain. 
If one aims to obtain a correct threshold law for the Coulomb system, then the interaction between the fragments, i.e. the particle correlation is to be taken into account. This makes the phase-space arguments insufficient, but requires dynamical treatment as it was originally done by Wannier [1] for the simplest system. In this paper we employ the most simple theoretical apparatus presenting the essential equations in an arbitrary coordinate frame. They remain valid, in particular, in the simplest single-particle Cartesian coordinates.

As discussed in Section [V], Poelstra et al [30] suggested another formula for the Wannier index which differs from our Eq.(54) by the extra "phase factor" $(N-2)$. This discrepancy remains hidden when one restricts consideration to the case of two, three, or four electrons receding from the positively charged core. In these cases the unstable mode proves to be respectively non-degenerate, doubly- and triply-degenerate. Thus the degree of degeneracy in these cases coincides with $(N-2)$. This fortuitously allows one to replace the summation over degenerate modes implied by formula (54) by multiplication over the factor $(N-$ 2) which corresponds to the formula by Poelstra et al [30]. However this coincidence is accidental and misleading. It is broken, for instance, by variation of charges and masses of the constituent particles which violates SCs symmetry and hence lifts the modes degeneracy, or by considering larger numbers of particles $N$ (simply because possible degrees of degeneracy are restricted by properties of the point groups in 3D space). For five electrons receding from a charged core only doubly degenerate unstable modes were found above.

Physically it is clear that if the charge of the core $Z$ in the system $\mathrm{A}^{Z}+(N-1) e$ becomes bigger, then the interelectron correlations should become less important and the threshold law should approach the value obtained from the phase-space arguments, i.e. $\mu \rightarrow(N-2)$ as $Z \rightarrow \infty$. This conclusion is supported by all examples considered. Moreover in all these examples one can note that:

- the number of unstable modes accounting for their degeneracy (i.e. the number of terms in the sum (54)) is equal to $(N-2)$;

- each partial Wannier index $\mu_{k 0}(55)$ tends to unity from above as $Z$ increases. 
Apparent exception from the first rule is an emergence of an additional unstable mode in the plane $A^{+Z}+4 e \mathrm{SC}$ for $Z=1$. However, this $\mathrm{SC}$ provides $\mu$ larger than $3 \mathrm{D} \mathrm{SC}$ and therefore it does not govern the threshold behaviour. Note that although these properties are physically very natural, it is not clear if they can be proven rigorously from the first principles. An additional observation is that the electrons in SC tend to be distributed uniformly on the sphere, even when the corresponding perfectly symmetrical body does not exist (see five-electron case above). For large number of electrons in the field of the core several competing SC are found to produce very close threshold indices. Still, in all the cases considered the leading SC is found to be the three-dimensional one.

These results hopefully should hold if the electrons are replaced by other (possibly different) negatively charged particles. However, the situation changes drastically if one of the "electrons" is replaced by a particle of positive charge, for example, positron. It is essential that an additional repulsive Coulomb interaction appears in the system. If correlations are neglected then the cross section decreases exponentially as $E$ approaches threshold. One could expect that although the true threshold law retains a power character for all values of $Z$, it tends to mock the exponential behaviour by increasing of $\mu$ value [53]. This property holds for all positron-containing systems considered above. The threshold index increases with $Z$ quite slowly. In order to illustrate the later point quantitatively we cite results for $\mathrm{A}^{+Z}+2 e^{-}+e^{+}$system with very large values of $Z$ (cf. Sec. $\mathrm{VB}$ ): $\mu=9.4$ for $Z=50$ $\left(\alpha=5.60^{\circ}, \beta=37.2^{\circ}\right) ; \mu=11.6$ for $Z=100\left(\alpha=3.95^{\circ}, \beta=37.8^{\circ}\right)$. In general terms one can argue that a similar situation should arise when a system contains two or more positively charged particles and two or more particles with negative charge. Note that the properties of the partial Wannier indices $\mu_{k 0}$ are less straightforward: some of them could be less than unity and vary with $Z$ non-monotonically.

Large values of threshold indexes $\mu$ are unfavorable for an experimental observation of the threshold behaviour: close to the threshold the cross section proves to be too small to be observable, and for higher excess energies the intrinsic deviations from the threshold law become essential. An analysis of the energy domain where the threshold law holds is 
beyond the scope of this paper. Still, we can note that for the electron-impact ionization of atoms or for double photoionization this domain is limited to few $\mathrm{eV}$ above threshold (for quantitative treatment within the Wannier mechanism see Refs. [4] [12]). For the positronimpact ionization the applicability domain is even less [12] [13]. As argued by Ihra et al [18], an agreement with experimental data could be substantially improved if the interaction of different modes in the deviation from SC is taken into account. Possibly some procedure to assess for the mode interaction could be developed also for the multifragment system; the present development provides a necessary first step for more advanced approaches. One could note also that even very large threshold indices could (quite unexpectedly) be useful for constructing formulae of interpolation character as shown in the recent paper by Rost and Pattard [20].

\section{ACKNOWLEDGMENTS}

We are thankful to D.V.Dzuba for his help in numerical calculations and to O.I.Tolstikhin for useful comments. M.Yu.K. is appreciates support from the Australian Research Council. V.N.O. acknowledges partial support from the Russian Foundation for Basic Research under the grant 96-02-17023. This work was supported by the Australian Bilateral Science and Technology Collaboration Program. V.N.O. is grateful for hospitality of the staff of the School of Physics of UNSW where this work was carried out.

\section{APPENDIX A: EIGENVALUES OF V MATRIX CORRESPONDING TO ROTATIONS AND TRANSLATIONS IN TIME}

If the $N$-particle system is rotated as a whole over infinitisemal (time-independent) angle $\delta \varphi$ around the axis $\vec{\nu}$, then the particle coordinates receive increments

$$
\delta \vec{r}_{j}^{(\nu)}=\left(\vec{\nu} \times \vec{r}_{j}\right) \delta \varphi
$$

The form of Newtonian equations of motion 


$$
m_{j} \frac{d^{2} \vec{r}_{j}}{d t^{2}}=-\frac{\partial U}{\partial \vec{r}_{j}}
$$

remains invariant under rotations. This implies that

$$
m_{j} \frac{d^{2} \delta \vec{r}_{j}}{d t^{2}}=-\sum_{i=1}^{N} \frac{\partial^{2} U}{\partial \vec{r}_{i} \partial \vec{r}_{j}} \delta \vec{r}_{j},
$$

where $\delta \vec{r}_{j}=\delta \vec{r}_{j}^{(\nu)}$. For SC one can use Eqs. (A1) and (11) to get

$$
\frac{d^{2} \delta \vec{r}_{j}^{(\nu)}}{d t^{2}}=\frac{1}{\phi} \frac{d^{2} \phi}{d t^{2}} \delta \vec{r}_{j}^{(\nu)} .
$$

Bearing in mind that according to (18)

$$
\phi^{2} \frac{d^{2} \phi}{d t^{2}}=-\frac{\mathcal{Q}_{0}}{\mathcal{M}}
$$

and using definition (29), we finally obtain

$$
\frac{1}{m_{j}} \sum_{i=1}^{N} V_{j i} \delta \vec{r}_{i}^{(\nu)}=\frac{\mathcal{Q}_{0}}{\mathcal{M}} \delta \vec{r}_{j}^{(\nu)}
$$

which means that the grand vector $\delta \mathbf{r}^{(\nu)}$ is an eigenvector of the grand matrix $\mathbf{K} \mathbf{V}$ with the eigenvalue $\mathcal{Q}_{0} / \mathcal{M}$. Generally there are three eigenvectors corresponding to this eigenvalue, but for a linear SCs only two independent rotations are possible.

Consider now variation of the trajectory caused by shifting of time over an infinitesimal interval $t \rightarrow t+\delta t$ using similar technique. For the system in $\mathrm{SC}$ the particle coordinates are incremented in this case by

$$
\delta \vec{r}_{j}^{(\mathrm{SC})}=\vec{v}_{j} \delta t=\frac{d \phi}{d t} \vec{\rho}_{j} \delta t
$$

The form of Newtonian equations of motion obviously remains invariant under the shift of the time variable. Therefore (A3) remains valid for $\delta \vec{r}_{j}=\delta \vec{r}_{j}^{(\mathrm{SC})}$. An analogue of Eq.(A4) now reads

$$
\frac{d^{2} \delta \vec{r}_{j}^{(\mathrm{SC})}}{d t^{2}}=\left(\frac{d \phi}{d t}\right)^{-1} \frac{d^{3} \phi}{d t^{3}} \delta \vec{r}_{j}^{(\mathrm{SC})}
$$

Differentiating Eq.(A5) we obtain 


$$
\frac{d^{3} \phi}{d t^{3}}=\frac{2 \mathcal{Q}_{0}}{\mathcal{M}} \frac{1}{\phi^{3}} \frac{d \phi}{d t}
$$

which finally brings us to

$$
\frac{1}{m_{j}} \sum_{i=1}^{N} V_{j i} \delta \vec{r}_{i}^{(\mathrm{SC})}=-\frac{2 \mathcal{Q}_{0}}{\mathcal{M}} \delta \vec{r}_{j}^{(\mathrm{SC})}
$$

Since the grand vector $\delta \mathbf{r}^{(\mathrm{SC})}$ is proportional to the grand vector $\boldsymbol{\rho}$ which defines the SC shape, we conclude that the latter vector is an eigenvector of the grand matrix $\mathbf{K ~} \mathbf{V}$ with the eigenvalue $-2 \mathcal{Q}_{0} / \mathcal{M}$ 


\section{REFERENCES}

* Permanent address: Institute of Physics, The University of St Petersburg, 198904 St.Petersburg, Russia; E-mail: Valentin.Ostrovsky@pobox.spbu.ru

[1] G. H. Wannier, Phys. Rev. 90, 817 (1953).

[2] S. Watanabe, J. Phys. B 24, L39 (1991).

[3] R. I. Hall, A. G. McConkey, L. Avaldi, K. Ellis, M. A. MacDonald, G. Dawber, G. C. King, J. Phys. B 25, 1195 (1992).

[4] A. K. Kazansky and V. N. Ostrovsky, J. Phys. B 25, 2121 (1992).

[5] X. Q. Guo and M. S. Lubell, J. Phys. B 26, 1221, 4885 (1993); M. S. Lubell, Z. Phys. D 30, 79 (1994); Nucl. Inst. Met. B 99, 177 (1995); Can. J. Phys. 74, 713 (1996).

[6] A. K. Kazansky and V. N. Ostrovsky, Phys. Rev. A 48, R871 (1993); J. Phys. B 26, 2231 (1993).

[7] M. S. Dimitriević, P. Grujić, and N.S.Simonović, J. Phys. B 27, 5717 (1994); W. Ihra, F. Mota-Furtado, and P. F. O’Mahony, Phys. Rev. A 55, 4263 (1997).

[8] A. K. Kazansky and V. N. Ostrovsky, Journal de Physique IV (Colloque C6; Suppl.JP II) 3, 159 (1993); J. Phys. B 27, 447 (1994); 28, 1453 (1995); 28, L333 (1995); Phys. Rev. A 51, 3712 (1995); 51, 3698 (1995); 52, 1175 (1995); Few-Body Systems Suppl. 8, 80 (1995); P. O. Bogdanovich, A. K. Kazansky and V. N. Ostrovsky, J. Phys. B 30, 921 (1997).

[9] J. Macek and S. Yu. Ovchinnikov, Phys. Rev. A 49, R4273 (1994); 50, 468 (1994).

[10] N. S. Simonović, Phys. Rev. A 50, 4390 (1994).

[11] A. K. Kazansky, V. N. Ostrovsky and L. Yu. Sergeeva. J. Phys. B 27, 5197 (1994).

[12] A. K. Kazansky, V. N. Ostrovsky, and L. Yu. Sergeeva, Z. Phys. D 33, 181 (1995). 
[13] P. Ashley, J. Moxom, and G. Laricchia, Phys. Rev. Lett. 77, 1250 (1996);

[14] S. Watanabe and D. Kato, J. Phys. B 29, L779 (1996); S. Watanabe, D. Kato, and M. Matsuzawa, Comments At. Mol. Phys. 33, 95 (1996); D. Kato and S. Watanabe, Phys. Rev. A 56, 3686 (1997).

[15] J. Macek and W. Ihra, Phys. Rev. A 55, 2024 (1996); W. Ihra, F. Mota-Furtado, P. F. O’Mahony, and J. H. Macek, Phys. Rev. A 55, 3250 (1997).

[16] A. K. Kazansky and V. N. Ostrovsky, In: 5th International Workshop 'Autoionization Phenomena in Atoms, Ed.V. V. Balashov, A. A. Grum-Grzhimailo, E. A. Romanovsky, Moscow University Press, 1996, pp.67-71.

[17] M. Yu. Kuchiev, J. Phys. B 30, 3499 (1997).

[18] W. Ihra, J. Macek, F. Mota-Furtado, and P. F. O’Mahony, Phys. Rev. Lett. 78, 4027 (1997);

[19] J. M. Feagin, and M. J. Goddard, J. Phys. B 30, 693 (1997).

[20] J. M. Rost and T. Pattard, Phys. Rev. A 55, R5 (1997).

[21] P. Grujić, Com. At. Mol. Phys. 33, 351 (1997).

[22] H. Klar and W. Schlecht, J. Phys. B 10, 1699 (1976).

[23] Some important details of the derivation were omitted, especially expression of the interaction potential in terms of new coordinates. The hyperspherical approach was not applied to multifragment positron containing systems.

[24] P. Grujić, J. Phys. B 16, 2567 (1983).

[25] P. Grujić, Fizika 15, 213 (1983).

[26] P. Grujić, Phys. Lett. A 96, 233 (1983); 122, 494 (1987).

[27] M. S. Dmitriević, P. Grujić, and N. Simonović Z. Phys. D 15, 203 (1990). 
[28] J. A. R. Samson and G. C. Angel, Phys. Rev. Lett. 61, 1584 (1988); see also H. Lebius, H. R. Kozlowski, K. Wiesemann, and B. A. Huber, Annalen der Physik (Leipzig) 48, $103(1991)$.

[29] J. M. Feagin and R. D. Filipczyk, Phys. Rev. Lett. 64, 384 (1990).

[30] K. A. Poelstra, J. M. Feagin, and H. Klar, J. Phys. B 27, 781 (1994).

[31] R. Stevens and J. M. Feagin, Bull. Am. Phys. Soc. 33, 992 (1988).

[32] H. Bluhme, H. Knudsen, and J. Merrison, In: XX International Conference on the Physics of Electronic and Atomic Collisions, edited by F. Aumayr, G. Betz and HP. Winter, Vienna 1997, p.TH 087.

[33] N. S. Simonović and P. Grujić, J. Phys. B 20, 3427 (1987).

[34] A. K. Kazansky and V. N. Ostrovsky, Zh. Eksp. Teor. Fiz. 95, 1162 (1989) [Sov. Phys. - JETP 68, 670 (1989)].

[35] A. K. Kazansky and V. N. Ostrovsky, In: Second International Workshop on Harmonic Oscillators, Ed.D.Han and K.B.Wolf, NASA Conference Publications 3286, NASA 1995, pp.349-358.

[36] U. Fano, Phys. Rev. A 24, 2402 (1981).

[37] U. Fano, Rep. Progr. Phys. 46, 97, (1983).

[38] R. K. Peterkop, J. Phys. B 4, 513 (1971).

[39] A. R. P. Rau, Phys. Rev. A 4, 207 (1971).

[40] T. Morishita, O. I. Tolstikhin, S. Watanabe, and M. Matsuzawa, Phys. Rev. A 56, 3559 (1997).

[41] J. M. Feagin, J. Phys. B 17, 2433 (1984).

[42] Note that this reasoning applies only to unstable modes. For the stable modes (which 
are responsible for particle angular correlation) the situation is drastically different, as discussed in detail in Ref. [6]; see also Ref. [8].

[43] C. H. Greene and A. R. P. Rau, J. Phys. B 16, 99 (1983); R. Peterkop, J. Phys. B 16, L587 (1983).

[44] S. Wolfram, Mathematica: A System for Doing Mathematics by Computer, 2nd ed. (Addison-Wesley Publishing Co., Palo Alto, 1991).

[45] H. Klar, J. Phys. B 14, 4165 (1981); H. Klar, In: Electronic and Atomic Collisions, Invited papers, XIII International Conference on the Physics of Electronic and Atomic Collisions, pp.767-775, Edited by J. Eichler, I. V. Hertel and N. Stolterfoht, Amsterdam, Elsevier, 1984.

[46] H. Klar, Z. Phys. A 307, 75 (1982).

[47] P. Grujić, J. Phys. B 15, 1913 (1982).

[48] It is worth mentioning that in variance with Klar and Schlecht we obtain correct value of $\mu$ without appealing to the contributions from undetermined angular variables $\psi$ and $\varphi$, see p.1709 of Ref. [22].

[49] The SCs $L_{b}$ and $P$ for this system were discussed originally by Grujić 25. However the configuration $P$ was discarded and the threshold indices evaluated for $L_{b}$ differ from these reported in the present paper.

[50] H. Kossmann, O. Schwarzkopf, B.Kämmerling, and V.Schmidt, Phys. Rev. Lett. 63, 2040 (1989);

[51] S. Geltman, J. Phys. B 16, L525 (1983).

[52] N. C. Sil and K. Roy, Phys. Rev. A 54, 1360 (1996).

[53] For the particular case of three-particle system the increase of $\mu$ with $Z$ was noticed by Klar [45]. 


\section{TABLES}

TABLE I. Parameters of scaling configurations and Wannier indices for $\mathrm{A}^{+Z}+2 e^{-}+e^{+}$system.

\begin{tabular}{|c|c|c|c|c|}
\hline$Z$ & SC parameters & $\mu_{1}$ & $\mu_{2}$ & $\mu$ \\
\hline \multicolumn{5}{|c|}{$\mathrm{SC} L_{a}$} \\
\hline 1 & $x=0.506100, \quad y=1.692952$ & 4.442178 & 2.193945 & 6.636123 \\
\hline 2 & $x=0.587468, \quad y=1.636629$ & 4.767141 & 2.064237 & 6.831377 \\
\hline 3 & $x=0.633155, \quad y=1.609587$ & 5.024502 & 1.966884 & 6.991386 \\
\hline 4 & $x=0.664214, \quad y=1.594313$ & 5.242328 & 1.88483 & 7.127158 \\
\hline \multicolumn{5}{|c|}{$\mathrm{SC} L_{b}$} \\
\hline 1 & $x=0.441380, \quad y=-0.677611$ & 2.577720 & 1.025435 & 3.603155 \\
\hline 2 & $x=0.539724, \quad y=-0.847969$ & 2.888492 & 1.009213 & 3.897705 \\
\hline 3 & $x=0.594480, \quad y=-0.949091$ & 3.193559 & 1.005040 & 4.198599 \\
\hline 4 & $x=0.631720, \quad y=-1.023071$ & 3.475766 & 1.003244 & 4.479010 \\
\hline \multicolumn{5}{|c|}{$\mathrm{SC} P$} \\
\hline 1 & $2 \alpha=76.7338^{\circ}, \quad 2 \beta=55.1969^{\circ}$ & 1.884950 & 1.562234 & 3.447184 \\
\hline 2 & $2 \alpha=55.1741^{\circ}, \quad 2 \beta=61.3793^{\circ}$ & 2.045028 & 1.793101 & 3.838128 \\
\hline 3 & $2 \alpha=45.4233^{\circ}, \quad 2 \beta=64.1787^{\circ}$ & 2.206553 & 1.972092 & 4.178645 \\
\hline 4 & $2 \alpha=39.5138^{\circ}, \quad 2 \beta=65.8916^{\circ}$ & 2.351217 & 2.123469 & 4.474686 \\
\hline
\end{tabular}


TABLE II. Wannier indices for $\mathrm{A}^{+Z}+4 e$ system. The numbers in parentheses indicate degree of unstable mode degeneracy.

\begin{tabular}{|c|c|c|c|c|}
\hline$Z$ & $\mu_{1}$ & $\mu_{2}$ & $\mu_{3}$ & $\mu$ \\
\hline \multicolumn{5}{|c|}{$\mathrm{SC} P$} \\
\hline 1 & 4.877419 & $4.248225(2)$ & 2.071837 & 15.44571 \\
\hline 2 & 1.356093 & $1.273381(2)$ & - & 3.902855 \\
\hline 3 & 1.192808 & $1.145660(2)$ & - & 3.484128 \\
\hline 4 & 1.132414 & $1.099316(2)$ & - & 3.331046 \\
\hline 5 & $1.100871(2)$ & 1.075346 & - & 3.251563 \\
\hline \multicolumn{5}{|c|}{$\mathrm{SC} V$} \\
\hline 1 & $3.075960(3)$ & - & - & 9.227870 \\
\hline 2 & $1.257986(3)$ & - & - & 3.773958 \\
\hline 3 & $1.139795(3)$ & - & - & 3.419384 \\
\hline 4 & $1.095940(3)$ & - & - & 3.287819 \\
\hline 5 & $1.073040(3)$ & - & - & 3.219120 \\
\hline
\end{tabular}


TABLE III. Parameters of SCs and Wannier indices for $\mathrm{A}^{+Z}+3 e^{-}+e^{+}$system. The numbers in parentheses indicate degree of unstable mode degeneracy.

\begin{tabular}{|c|c|c|c|c|c|c|}
\hline$Z$ & SC parameters & & $\mu_{1}$ & $\mu_{2}$ & $\mu_{3}$ & $\mu$ \\
\hline \multicolumn{7}{|c|}{$\mathrm{SC} L$} \\
\hline 1 & $x=0.580448, \quad y=-1.070391$ & $z=1.627861$ & 4.41213 & 2.30976 & 1.06890 & 7.79079 \\
\hline 2 & $x=0.580448, \quad y=-1.070391$ & $z=1.627861$ & 4.73844 & 2.13221 & 1.03343 & 7.90408 \\
\hline 3 & $x=0.628772, \quad y=-1.162883$ & $z=1.602043$ & 4.99954 & 2.01766 & 1.02270 & 8.03998 \\
\hline 4 & $x=0.661096, \quad y=-1.22438$ & $z=1.587485$ & 5.21972 & 1.92598 & 1.01746 & 8.16316 \\
\hline \multicolumn{7}{|c|}{$\mathrm{SC} V$} \\
\hline 1 & \multicolumn{2}{|c|}{$\alpha=60.5698^{\circ}, \quad \beta=32.2041^{\circ}$} & $1.57584(2)$ & 1.03194 & $0.60493(2)$ & 5.39348 \\
\hline 2 & \multicolumn{2}{|c|}{$\alpha=40.5400^{\circ}, \quad \beta=41.7154^{\circ}$} & $1.56354(2)$ & 1.20043 & $0.66302(2)$ & 5.65356 \\
\hline 3 & \multicolumn{2}{|c|}{$\alpha=32.3675^{\circ}, \quad \beta=44.9869^{\circ}$} & $1.70957(2)$ & 1.33711 & $0.62771(2)$ & 6.01166 \\
\hline 4 & \multicolumn{2}{|c|}{$\alpha=27.6668^{\circ}, \quad \beta=46.7663^{\circ}$} & $1.85129(2)$ & 1.45098 & $0.57327(2)$ & 6.30011 \\
\hline
\end{tabular}


TABLE IV. Parameters of SCs and Wannier indices for $\mathrm{A}^{+Z}+5 e$ system. The numbers in parentheses indicate degree of unstable mode degeneracy.

\begin{tabular}{|c|c|c|c|c|c|}
\hline$Z$ & SC parameters & $\mu_{1}$ & $\mu_{2}$ & $\mu_{3}$ & $\mu$ \\
\hline \multicolumn{6}{|c|}{$\mathrm{SC} P$} \\
\hline 2 & - & $1.818250(2)$ & $1.575289(2)$ & $0.701595(2)$ & 6.787079 \\
\hline 3 & - & $1.363938(2)$ & $1.245279(2)$ & - & 5.218433 \\
\hline 4 & - & $1.235701(2)$ & $1.156156(2)$ & - & 4.783715 \\
\hline 5 & - & $1.174520(2)$ & $1.114540(2)$ & - & 4.578120 \\
\hline 6 & - & $1.138614(2)$ & $1.090432(2)$ & - & 4.458093 \\
\hline 7 & - & $1.114982(2)$ & $1.074705(2)$ & - & 4.379373 \\
\hline \multicolumn{6}{|c|}{$\mathrm{SC} V$} \\
\hline 2 & $\alpha=45.15762^{\circ}$ & 1.606923 & 1.504688 & $1.493106(2)$ & 6.097823 \\
\hline 3 & $\alpha=45.09672^{\circ}$ & 1.280163 & 1.228075 & $1.223717(2)$ & 4.955672 \\
\hline 4 & $\alpha=45.06976^{\circ}$ & 1.182908 & 1.147576 & $1.145087(2)$ & 4.620659 \\
\hline 5 & $\alpha=45.05455^{\circ}$ & 1.135887 & 1.109091 & $1.107406(2)$ & 4.459790 \\
\hline 6 & $\alpha=45.04479^{\circ}$ & 1.108127 & 1.086528 & $1.085274(2)$ & 4.365202 \\
\hline 7 & $\alpha=45.03799^{\circ}$ & 1.089794 & 1.071698 & $1.070708(2)$ & 4.302909 \\
\hline
\end{tabular}

TABLE V. Parameters of SCs and Wannier indices for $2 X_{m}^{Z}+2 e$ system. The numbers in parentheses indicate degree of unstable mode degeneracy.

\begin{tabular}{|c|c|c|c|c|c|c|}
\hline$Z$ & $m$ & $\alpha$ & $\mu_{1}$ & $\mu_{2}$ & $\mu_{3}$ & $\mu$ \\
\hline 1 & 1 & $\alpha=45^{\circ}$ & 1.29366 & $0.90584(2)$ & - & 3.10533 \\
\hline 2 & 1 & $\alpha=32.2093^{\circ}$ & 1.36762 & 1.33643 & - & 2.70405 \\
\hline 1 & 2 & $\alpha=35.9490^{\circ}$ & 1.56958 & 1.31788 & 0.54315 & 3.43062 \\
\hline 1 & 1836 & $\alpha=30.0049^{\circ}$ & 50.32979 & 37.46232 & - & 87.79211 \\
\hline
\end{tabular}

